\title{
Lessons from the Inaugural Satellite Design Challenge
}

\author{
Dario Schor ${ }^{1}$, Kane Anderson ${ }^{1}$, Ahmad Byagowi ${ }^{1}$, Brady Russell ${ }^{1}$, Scott McKay ${ }^{2}$, Veronica Marriott ${ }^{3}$, \\ Reza Fazel-Darbandi ${ }^{l}$,Matthew Woelk, Anthony Schoenfeld ${ }^{3}$, Craig Nemeth ${ }^{l}$, Pawel Glowacki ${ }^{l}$, \\ Greg Linton ${ }^{1}$, Witold Kinsner ${ }^{1}$, M.D. (Ron) Britton ${ }^{4}$, and Malcolm Symonds ${ }^{4}$ \\ ${ }^{1}$ Dept. of Electrical \& Computer Engineering, ${ }^{3}$ Dept. of Mechanical Engineering, ${ }^{4}$ Faculty of Engineering \\ ${ }^{2}$ Dept. of Physics \& Astronomy, Faculty of Science \\ University of Manitoba, Winnipeg, MB, Canada R3T 5V6 \\ dario_schor@umanitoba.ca, witold.kinsner@ad.umanitoba.ca
}

\begin{abstract}
The inaugural Canadian Satellite Design Challenge provided many experiential learning opportunities for students through the design, implementation, and testing of the T-Sat1 nanosatellite. The University of Manitoba team elected to build all components from the ground up in order to develop many technical and non-technical skills that complement the classroom experience.

The first phases of the project focused on the definition of requirements, specifications, and interfaces. Then, the team began prototyping, implementing, and integrating subsystems. Finally, the spacecraft was tested at the Canadian Space Agency laboratories in Ottawa and obtained second place overall in the competition.

This paper describes some of the major lessons from the implementation, integration, and testing phases of the project. In addition, some insight is provided as to the ongoing changes for the second iteration of the competition that aim at improving the experience and performance of the satellite.
\end{abstract}

Keywords: Canadian Satellite Design Challenge, tripepicosatellite, complex systems.

\section{INTRODUCTION}

The Canadian Satellite Design Challenge (CSDC) was introduced in 2010 to expand experiential learning of undergraduate and graduate students with respect to complex systems [Geoc09]. In this competition, thirteen university teams attempted to designed, built, and tested an operational nanosatellite (code T-Sat1), while also managing the project, securing the necessary funding, and reaching out to the community. The project expands classroom learning through a holistic experience that introduces students to multidisciplinary teams, budgets, timelines, legalities, feasibility studies, trade-off analysis, risk management, and long-term projects.

The University of Manitoba team that competed in the challenge consisted of more than 100 undergraduate and graduate students from Engineering, Science, Business,
Architecture, and Art working together in groups on the T-Sat1 project [KBSF11] [KSFC13]. The students worked closely with more than 50 advisors that provided feedback on the activities. The advisors came from academia, industry, business, military, and government thus providing wide variety of perspectives on the project. The two-year T-Sat1 project concluded in September 2012, when eleven students from the University of Manitoba tested the satellite at the Canadian Space Agency laboratories in Ottawa, and obtained second place overall in the inaugural competition.

Throughout the two-year competition, the team faced many technical and non-technical challenges. Most of the technical challenges dealt with the interdependent nature of the designs that forced students to evaluate their ideas from a different perspective (i.e., the printed circuit board layout no longer needed to be functional, but also placed tall integrated chips on certain locations in order to have a good mechanical fit with other components). The nontechnical challenges included working in a large and multidisciplinary team, managing long-term project deadlines, keeping students motivated, dealing with student changeover, and reasoning through evolving solutions.

This paper presents some of the lessons learned from the first iteration of the competition at the University of Manitoba. Many of these actions are currently being implemented for the second iteration of the competition with good results.

\section{T-SAT1 OVERVIEW}

T-Sat1 is designed for a Sun-synchronous orbit with an altitude in the range of $600-800 \mathrm{~km}$ above the ground [Geoc11a]. Simulations revealed that it would orbit the Earth every 90-96 minutes and only have contact with the University of Manitoba Satellite Ground Station [ScKT09] for approximately 30 minutes per day spread through a 4-5 passes with a maximum time of 9 minutes.

The satellite hosts two on-board experiments and all the necessary subsystems to support these operations. It is $10 \times 10 \times 34 \mathrm{~cm}^{3}$ (as shown on Fig. 1), and has a maximum 


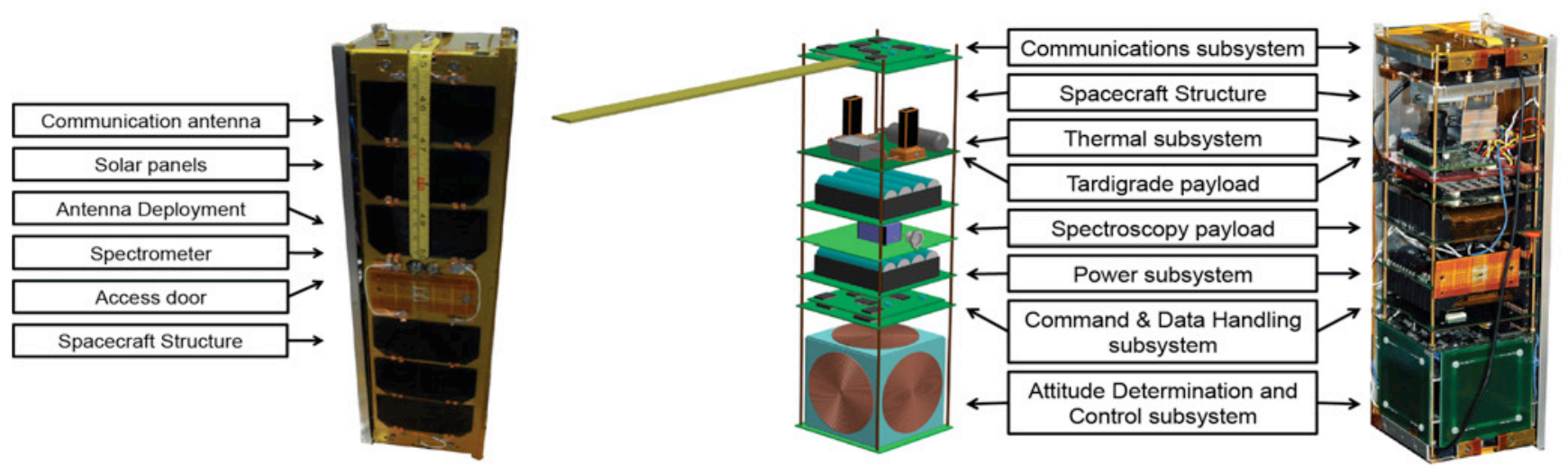

Fig. 1. Photograph of T-Sat1 (left), SolidWorks model (middle), and photograph of T-Sat1 components (right).

mass of $4 \mathrm{~kg}$ (actual $2.5 \mathrm{~kg}$ ). The following sections describe the scientific payloads and the subsystems that make up the satellite.

\subsection{Science Payloads}

The two science experiments in T-Sat1 are designed to address important scientific experiments while also challenging students in the design, implementation, and testing phases of the project.

Tardigrade Experiment: The low-cost astro-biological platform addresses the question of where else life might exist and whether life can be transported through space on meteors [MFSG12]. The test places a colony of tardigrades and monitoring their behavior when exposed to the harsh space environment. To make this possible, the tardigrades are launched in a cryptobiotic state and revived in space with nutrients and water.

Spectroscopy Experiment: This experiment aims to demonstrate that spectroscopy at the nanosatellite scale [FMSG12]. More specifically, a miniature spectrometer is used to measure solar phenomena that affect total energy output at specific wavelengths. The results are compared to those of larger missions on the ground to validate the performance of the system.

\subsection{Spacecraft Bus}

The following six subsystems in the spacecraft are design to support the payload experiments and satisfy the mission requirements.

Attitude Determination and Control (ADC) subsystem: This subsystem controls is responsible for stopping the post-launch tumbling motion and then orienting the satellite for the spectroscopy payload. The subsystem hardware senses the environment to determine the current orientation using a magnetometer and photodiodes on all faces of the spacecraft. The actuators consist of custom magnetic torque discs described in [WEKF12] and [RCHB13]. The software portion is a combination of the B-Dot algorithm for detumbling, and a sensor-fusion algorithm [RFHN12] for Sun-pointing.

Communications (COM) subsystem: This subsystem allows the satellite to transmit telemetry and receive commands from the University of Manitoba Satellite Ground Station [ScKT09] using amateur radio bands. The hardware consists of a commercial-off-the-shelf (COTS) transceiver from Yaesu and a custom terminal-nodecontroller to process the digital data [AWNA12]. Two tape-measure antennas are used to obtain quality communications under the extreme conditions expected in orbit [KSFC13].

Command and Data Handling (CDH) subsystem: The subsystem controls the operations of the spacecraft. It uses an MSP430 microprocessor and some additional peripherals. The flight software schedules tasks to meet the mission requirements while balancing the limited resources on the system (i.e., power, communication time, and payload requirements) [KSFC13].

Power (PWR) subsystem: The subsystem collects energy from the Sun through solar panels and stores it in batteries that power the spacecraft operations [FDTA12]. Additional hardware is used to distribute power throughout the satellite and protect the individual subsystems.

Thermal (THM) subsystem: An active heater is used to keep the water reservoir for the tardigrades from freezing under the extreme temperatures of low-Earth-orbit [CMSM12]. Additional sensors are used throughout the spacecraft to monitor the temperature of the electronics, however finite-element-analysis predicts that these will remain within their operating ranges due to the excess 
heat from the processors and other key components in the satellite.

Structure (STR) subsystem: The structure houses all the components of the satellite and protects it from the $12 \mathrm{~g}$ forces experienced during launch. It is designed of Aluminium 6061-T6 and conforms to the P-POD launcher requirements [CMSM12] [Cube01].

\section{IMPLEMENTATION AND INTEGRATION}

The implementation phase consisted of small subsystem prototypes transitioning into a finish product that could be integrated in the satellite. The process required lots of multidisciplinary collaboration to fit all the components in the small space. The following paragraphs describe some of the key lessons learned throughout the process.

\subsection{Electrical Components}

The implementation of electrical components consists of breadboard implementations to verify the circuit designs before producing a printed circuit board (PCB). The main challenge in this process was incorporating mechanical constraints into the finished PCBs such that they would fit in the spacecraft. Most subsystems had to adhere to a well-defined cubic volume, however, the COM and PWR subsystems had unique height profiles to adhere to in order to fit the components. This created many challenges and extra iterations to find an ideal placement on the boards.

In addition, the PCB design included many access ports connections, test points, and means of soldering connections to components not mounted on boards (i.e., torque discs, solar panels, and photodiodes). In this process, the team failed to account for the difficulty in soldering and accessing these components during the integration phase and testing, as shown in Fig. 2. As a result, some of the wires connecting solar panels and torque discs were longer than originally planned because it was impossible to reach these devices otherwise during the assembly. Furthermore, this made some components inaccessible for debugging or testing without having to resolder connections. The best last minute solution tested was to use small wire-to-wire connectors and was successfully implemented for a small critical portion of the connectors.

Similarly, the PC-104 bus is used to interconnect subsystems as it had proved successful for other cubesat missions. However, the team found this bus very difficult to work with in the triple-cubesat configuration as debugging or modifying pieces on certain subsystems required disassembling the entire structure. Instead, backplane buses (i.e., PCI interfaces on PCs) or other configuration not involving threaded rods to hold the boards need to be considered in future iterations.

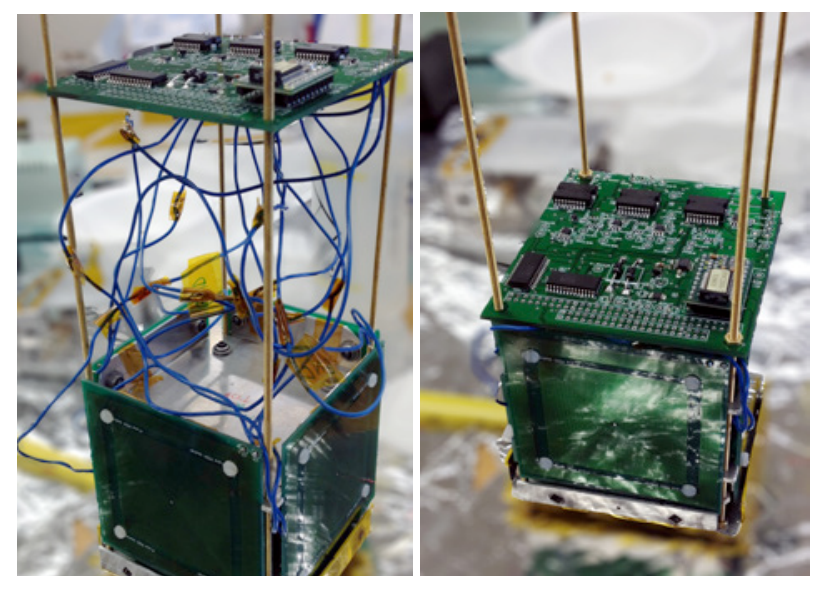

Fig. 2. Soldering cables from the torque discs to the ADC driver board (left) and finished product (right).

\subsection{Mechanical Components}

The mechanical challenges included managing the tight space and center of mass for the spacecraft. The mass and size of major components (not including passive surface-mount devices) was modeled using SolidWorks prior to the integration and updated as needed throughout the integration process. The result distributed the batteries into two places surrounding the spectroscopy experiment and mounted the tardigrades and ADC subsystems on opposite ends to account for the large open air pieces that affected the mass distribution. The analysis of the volume revealed details that helped placed components. In addition, this revealed the need for grooves on the edges of the PCBs to account for some wires running along the sides of the spacecraft.

The structure for the satellite evolved through many iterations in the two year project. Initially, it consisted of a rib-like structure that would connect all the components, however it was found difficult to implement to the required tolerances given the limited time and resources. After many variations, the structure settled into a threaded rod assembly to hold the PCBs and connect to a top, middle, and bottom Aluminum pieces that connected to the outside shell. The shell was intended to be a solid piece that could slide over the entire spacecraft, but was modified into two pieces that facilitated accessing components. Many last minute adjustments to the structure were required as one axis was manufactured incorrectly and was $1.8 \mathrm{~mm}$ bigger than expected and the top plates were folded incorrectly requiring some last minute adjustments to fit the antennas. 


\section{TRANSPORTING THE SPACECRAFT}

Packaging and transporting the spacecraft from the University of Manitoba to the David Florida Labs in Ottawa required special consideration given the delicate components and strict timelines that had the team working until the last possible minute.

\subsection{Packing T-Sat1}

The spacecraft solar panels were assembled in a cleanroom facility and needed special care to prevent dust particles from adhering to the surfaces and affecting their performance. To address this, the team placed the entire satellite in protective cleanroom plastic bags. The bag with the spacecraft was placed in a solid suitcase with protective foam. Although this was effective, the process can be improved for future iterations by separating the faces of the satellite with solar panels and packing them separately. This would not only make it easier to protect the devices from dust and damage, but also facilitate the challenges with transporting the satellite described in the next section.

\subsection{Transporting T-Sat1}

Three options were considered for transporting the spacecraft from the University of Manitoba to the David Florida Laboratories.

The first option was to work with Magellan Aerospace to ship the spacecraft directly to the David Florida Laboratories like they do for their spacecraft. Although this was the preferred option, it was not feasible because it required at least two weeks to ensure it was properly packaged, shipped, received, and processed at both ends. Alternatively, the spacecraft could be shipped through commercial companies overnight and received at the hotel in Ottawa, however, even with insurance, this was not a risk the students were willing to take.

The second option was to fly with the spacecraft to Ottawa. The difficulty with this option was dealing with airport authorities and the security procedures. Extensive conversations with representatives from the Winnipeg James Armstrong Richardson International Airport, the Ottawa Macdonald-Cartier International Airport, and Transport Canada revealed that upon reaching security the officer would pass the spacecraft through the x-ray machine and then had the option to inspect the device if he thought it was necessary. The inspection would require taking the spacecraft out of the box, out of the plastic cleanroom bags, and possibly opening it to show the batteries inside. This could contaminate the solar panels and possibly damage other pieces. If the team refused to take the spacecraft out of the cleanroom bags, the passengers could be asked to go to a private security room for interrogation and risk missing the flight. Having worked hard for two years, the team felt the risk of missing environmental testing for airport security was not deemed acceptable.

The final option was to drive to Ottawa with the spacecraft. The risks here were a long drive with the satellite in a suitcase. This was deemed to be the best option that was within the student's control. The students carried letter from the University of Manitoba attesting the contents and the destination of the load carried.

For future iterations, the preferred method is to ship the spacecraft directly to Ottawa. Alternatively, special permits and separate packaging for pieces of the spacecraft could be used to be able to fly with the hardware and successfully go through airport security.

\section{ENVIRONMENTAL TESTING}

The CSDC planned for three tests at the David Florida Laboratories: (i) vibrations, (ii) thermal vacuum, and (iii) functional testing.

\subsection{Vibrations Testing}

The vibrations test consists of three runs exciting each axis of the spacecraft. The first run is a sinusoidal sweep from $1 \mathrm{~Hz}$ to $2,000 \mathrm{~Hz}$ that establishes the baseline response of the system to different frequencies and identifies the fundamental frequency (which must be greater than $90 \mathrm{~Hz}$ as per CSDC requirement [Geoc11b]). A 1-inch peak-to-peak displacement is experienced at all frequencies. The second run uses a controlled random vibration pattern that simulates a worst-case scenario for a launch vehicle. This stresses the components and manufacturing process to ensure that all systems are operational after launch. Finally, the sinusoidal sweep is performed again to compare the responses for each frequency pre- and post- random vibrations.

The experimental setup consists of many small accelerometers connected to the body of the spacecraft as shown in Fig. 3. For triple cubesats, a minimum number of sensors is placed along each axis, and then a few extra sensors are added to capture the response of some of the critical hardware on the spacecraft. Once the sensors are installed, the spacecraft is placed in an aluminum chamber that resembles the contact points for a Poly PicoOrbital Deployer (P-POD) launcher [Cube01]. The PPOD structure makes contact with the spacecraft along the rails and standoffs only, thus protecting all the exterior panels. The P-POD is then secured onto a slip plate to the UD-4000 Shaker table for the tests.

The team placed 2 accelerometers along each axis of the spacecraft body to capture the performance during the vibrations test. Additional sensors were placed on the tardigrade camera and spectrometer as these were designed using free-floating structures to absorb some of the vibrations and protect the imaging systems. The team 
experienced some difficulties placing the spacecraft in the $\mathrm{P}-\mathrm{POD}$ as one side was $1.8 \mathrm{~mm}$ bigger than the original design. However, once inside the P-POD, the tests proceeded as expected.

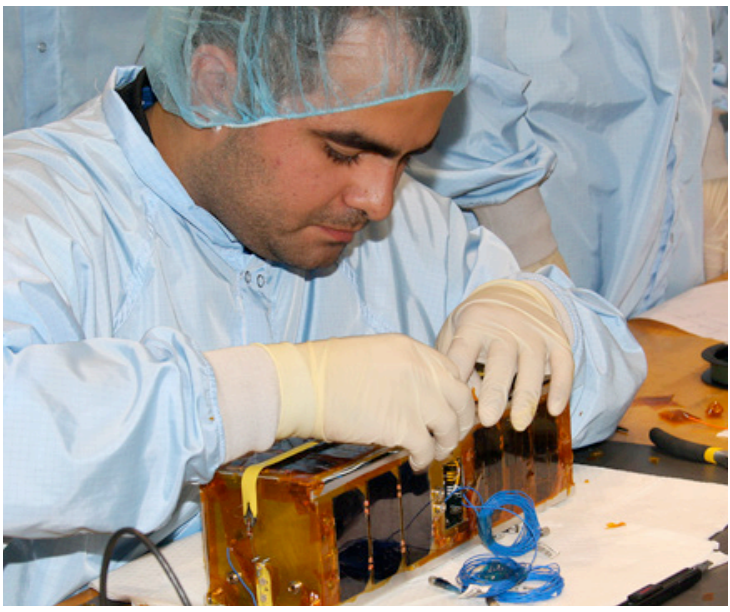

Fig. 3. Placing accelerometers to the satellite for vibrations testing. Photo credit: Canadian Space Agency.

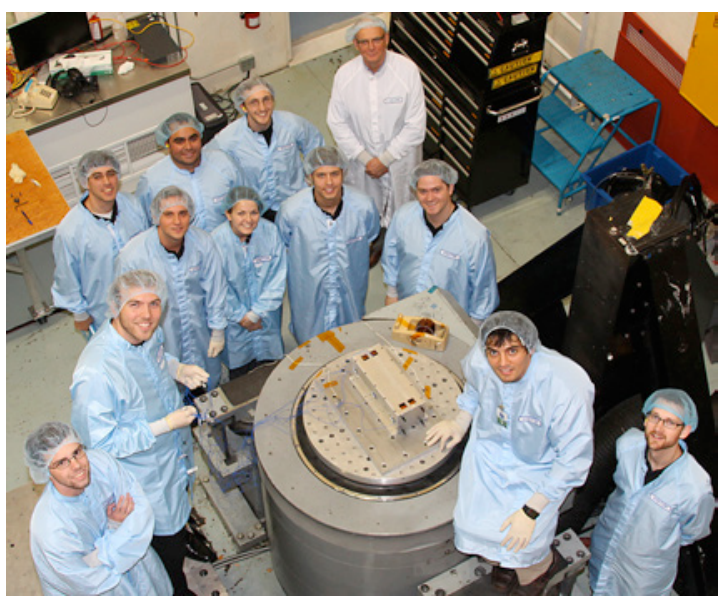

Fig. 4. Team members pose for a picture before starting the vibrations testing. The satellite is inside the P-POD mounted on the vibrations table in the middle of the picture. Photo credit: Canadian Space Agency.

The vibration results showed a fundamental frequency well above $90 \mathrm{~Hz}$ and within $5 \mathrm{~Hz}$ of the expected values from simulations. The main lessons learned was to plan for the sensor placement in advance in order to leave enough space to access the specific components (i.e., tardigrade chamber) and account for the size of the wires that lead from the accelerometer to the vibrations table hardware. Planning for the test phase would facilitate the test procedures.

\subsection{Thermal Vacuum Testing}

The thermal vacuum test simulates the space environment and is designed to verify that no components would outgas once in orbit. Although planned for CSDC, this test was cancelled for all teams as none could produce the required documentation to prove that all components used would not outgas and expel chemicals that could contaminate the Canadian Space Agency equipment. To overcome this in future projects, the team members are checking online databases from reputable sources to select components and plans to outgas components locally whenever there is a doubt.

Furthermore, it is common for industry satellites to be operational during the thermal vacuum tests to verify that all components can work at the extreme temperatures. This was not incorporated into the inaugural CSDC, but plans exist to require special harnesses such that the tests could be conducted following industry standards.

\subsection{Functional Testing}

Functional testing is designed to demonstrate that the spacecraft meets the requirements and it is operational. The demonstration is executed by powering up the satellite and letting some of the key initial tasks execute automatically as seen on Fig. 5. Then, specific commands could be issue to verify other working components.

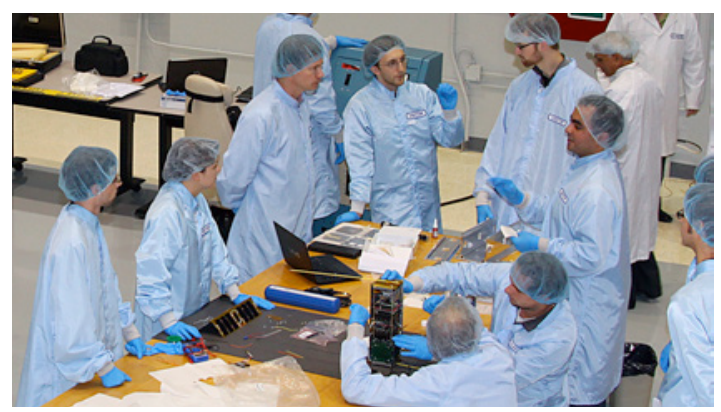

Fig. 5. Team members explaining and verifying results with the judges during functional testing. Photo credit: Canadian Space Agency.

The power-up sequence for T-Sat1 showed the two antennas deployed correctly. Then, a wire harness was connected to obtain payload data and verify that both the tardigrade camera and spectrometers were operational. Although command, telemetry, and other modes were to be demonstrated, some components were not fully operational and/or were difficult to test without a proper node to act as a ground station during testing. Time constraints caused all these tests to be compressed into a two-hour window leaving out some of the demonstrations planned. Instead the team members present showed some of the results captured in Winnipeg that demonstrated the 
ADC subsystem working and other pieces required for the competition.

For future missions, the team is developing specific test plans to execute and demonstrate the spacecraft is working. A laptop ground station connected to a handheld radio is being implemented to verify the operation.

\section{MAIN LESSONS LEARNED}

Having completed one iteration of the CSDC, there are three major lessons learned by the University of Manitoba team: (i) integrated design, (ii) documentation, and (iii) understanding the design phases.

As described in Sec. 3, there were many issues raised that could have been resolved with better collaboration between the electrical and mechanical teams. This problem is being addressed through internal technical standards for manufacturing, layouts, interfaces, and parts that aim to ensure the interconnections between subsystems are established early in the design process. Furthermore, the team structure was modified by creating a Systems Integration Lead position whose primary responsibility is looking at interfaces, how things will be connected, and plan for the manufacturing and testing. This is a very demanding position and one that requires a high degree of technical competency to achieve the objectives of the project.

Although many elements of the project are well documented through publications, internal documents, and a team wiki, some of the background work that lead to the decisions was not always captured. Just as important, the team lacked explicit procedures for testing that would reduce the uncertainty and facilitate the functional testing. In the second iteration of the CSDC, the team is making better use of the wiki and documenting key design decisions. Furthermore, the test plans are being developed in conjunction with the requirements to ensure that all components can be tested.

Finally, the phases of the design process during the first iteration of CSDC placed a lot of emphasis on the preliminary designs and had very little time allotted to the construction of the actual satellite [KBSF11][KSFC13]. This is modeled after the aerospace industry and was necessary to develop a body of knowledge of basic spacecraft design parameters. To complement this, the new satellite is encouraging a lot more hands-on experiential learning during early phases of the design to address the mixture of backgrounds from the students participating.

\section{CONCLUDING REMARKS}

The inaugural CSDC met the intended objectives of providing students with experiential learning opportunities in the aerospace sector. The experience of designing, building, and testing the spacecraft at the
Canadian Space Agency labs motivated students to develop many new skills and expand their education.

The University of Manitoba team made a conscious decision to build all subsystems from the ground up for the inaugural CSDC. The cost of this operation was that there was much time devoted to developing requirements and analyzing the designs. The cost of this decision was a relatively slower progress, rushed implementation, and a near-functioning spacecraft instead of a fully operational and space-ready satellite. The investment in a fully customized system provided depth into the experiential learning process for the students and advisors. This process enabled the team to develop a body of knowledge to build on for future iterations of the T-Sat project.

T-Sat1 served as the first prototype of a nanosatellite for the University of Manitoba. The experiential learning prepared the team for a new iteration that would be able to build on the experience by fixing and improving prototypes.

The educational objectives to expose students to complex systems and expand the classroom knowledge were achieved. In addition, T-Sat 1 made space projects accessible to undergraduate and graduate students. The complex project exposed students to technical and nontechnical skills, while also collaborating with many different professional groups and organizations. This collaboration has brought the groups much closer together in their awareness of complex projects at the University of Manitoba, while providing opportunities to the companies to observe potential employees in action.

\section{Acknowledgements}

Special thanks to Mr. Larry Reeves from Geocentrix for coordinating this exceptional educational experience.

This work could not have been completed without the immense effort and dedication from all the students and advisors for T-Sat1. In particular, thank you to Gord Klimenko for all his assistance during the integration phase. Special thanks to Diane Kotelko, Dr. Philip Ferguson, and Raymond Harris from Magellan Aerospace for their ongoing support throughout the project. Finally, thank you to Dwayne Chrusch from the Nano Systems Fabrication Laboratory at the University of Manitoba for his invaluable assistance with the solar panels.

Thank you also go to Dr. Alex Jablonski, Shantnu Mishra, Paul Marchand, Mark O'Grady, and Fred Sauve from the David Florida Labs for all their assistance and guidance during environmental testing.

This work was made possible thanks to the financial contributions from Magellan Bristol Aerospace Ltd., Analytical Graphics Inc., Emcore Corporation, Texas Instruments, and SolidWorks (Platinum level); University of Manitoba (UofM), UofM Engineering Endowment Fund, UofM Friends of Engineering, Alan D. and Phyllis 
Ann Thoren Fund (at the Winnipeg Foundation), Shell Canada, Standard Aero, IMRIS Inc., and MindSet/Manitoba Government (all Gold level), Parker Hannifin Corporation, IEEE Canadian Foundation, UofM Students' Union, Canadian Space Agency, Canadian Forces School of Aerospace Studies, Ocean Optics, Qioptics Space Technologies, Gamble Technologies, Wacker Chemie AG, Acryl Design Ltd., and Kinetic Machine Works Inc. (Silver level), as well as UofM Engineering Society (Bronze level).

\section{References}

[AWNA12] Kane Anderson, Matthew Woelk, Craig Nemeth, Hossain Al-Masroor, Greg Linton1, Frank Serafin, Johnson Vilayvanh, Dario Schor, Witold Kinsner, Diane Kotelko, and Alan Thoren, "Telemetry and Telecommand Architecture for a Nanosatellite," in Proc. 16th CASI Astronautics Conf., ASTRO12, (Quebec City; April 24-26, 2012).

[CMSM12] Brendan Cade, Morgan May, Anthony Schoenfeld, Raamjee Mailvaganam, Veronica Marriott, David Newsom, Dario Schor, Witold Kinsner, and Paul Labossiere, "Structural and thermal analysis for the T-Sat1 nanosatellite," in Proc. 16th CASI Astronautics Conf., ASTRO12, (Quebec City; April 24-26, 2012).

[Cube01] CubeSat Design Specifications Document, Revision 3. Palo Alto, CA: Stanford University and California Polytechnic Institute at San Louis Obispo. March 2001. Available as of July 15, 2011 from http://ssdl.stanford.edu/cubesat/

[FDTA12] Arash Fazel-Darbandi, Jesse Doerksen, Jason Tayler, Udeesha Annakkage, Kasun Samarasekera, Mohammadreza Fazel Darbandi, Pawel Glowacki, Dario Schor, Witold Kinsner, Diane Kotelko, Philip Ferguson, David Weber, and Samuel Kovnats, "Design and implementation of a power system for the T-Sat1 nanosatellites," in Proc. 16th CASI Astronautics Conf., ASTRO12, (Quebec City; April 24-26, 2012).

[FMSG12] Cody Friesen, Scott McKay, Graham Schellenberg, Ben Guest, Alex Paterson, Dario Schor, and Witold Kinsner, "Solar spectroscopy for the T-Sat1 nanosatellite," in Proc. 16th CASI Astronautics Conf., ASTRO12, (Quebec City; April 24-26, 2012).

[Geoc09] Geocentrix Technologies Ltd., H.R.MacMillan Space Centre, and Jaymie M. Matthews, A proposal to initiate a satellite design challenge for Canadian universities. Vancouver, BC: Geocentrix, January 13, 2009.
[Geoc11a] Geocentrix Technologies Ltd., CSDC Rules and Regulations: Mission-level requirement document. Vancouver, BC: Geocentrix, January, 2011.

[Geoc11b] Geocentrix Technologies Ltd., CSDC Rules and Requirements. Vancouver, BC: Geocentrix, January, 2011.

[KBSF11] Witold Kinsner, M.D. (Ron) Britton, Dario Schor, Arash Fazel-Darbandi, Kris Goodmanson, Cody Friesen, and Emily Bashford, "Preliminary experience from the Canadian Satellite Design Challenge," in Proc. Can. Engineering Education Conf. (St. John's, NL; June 6-8, 2011) 2011 .

[KSFC13] Witold Kinsner, Dario Schor, Reza Fazel-Darbandi, Brendan Cade, Kane Anderson, Cody Friesen, Scott McKay, Diane Kotelko, and Philip Ferguson, "The T-Sat1

Nanosatellite Design and Implementation through a Team of Teams," International Journal of Software Science and Computational Intelligence, pp. 1-17, 2013. (Accepted for publication)

[MFSG12] Scott McKay, Cody Friesen, Graham Schellenberg, Ben Guest, Alex Paterson, Dario Schor, and Witold Kinsner, "A prototype for a tardigrade experiment for the T-Sat1 mission," in Proc. 16th CASI Astronautics Conf., ASTRO12, (Quebec City; April 24-26, 2012).

[RCHB13] Brady Russell, Lee Clement, Joshua Hernandez, Ahmad Byagowi, Dario Schor, and Witold Kinsner, "Implementation of a nanosatellite attitude determination and control system for the T-Sat 1 mission," for the IEEE 2013 Conference on Electrical and Computer Engineering, CCECE 2013 (Regina, SK; May 5-8, 2013), 5 pp. (Accepted for publication)

[RFHN12] Brady Russell, Mohammadreza Fazel-Darbandi, Joshua Hernandez, David Newsom, Brendan Cade, Dario Schor, Witold Kinsner, Diane Kotelko, and Philip Ferguson "Implementation of attitude control algorithms for the TSat1 nanosatellite mission," in Proc. 16th CASI Astronautics Conf., ASTRO12, (Quebec City; April 24-26, 2012).

[ScKT09] Dario Schor, Witold Kinsner, and Alan Thoren, "Satellite ground station emulator: An architecture and implementation proposal," in Proc. of the IEEE Canadian Conference on Electrical and Computer Engineering, CCECE 2009, (St. John's, NL; May 3-6, 2009), 2009, pp. 874-879.

[WEKF12] Paul White, Trent Erskine, Shawn Koop, Marc Friesen, Kiral Poon, Brady Russell, Mohammadreza FazelDarbandi, Joshua Hernandez, David Newsom, Brendan Cade, Dario Schor, and Witold Kinsner, "Design and implementation of magnetic torque rods for the T-Sat1 nanosatellite mission," in Proc. 16th CASI Astronautics Conf., ASTRO12, (Quebec City; April 24-26, 2012). 